\title{
Numerical Simulations through First Order Nonlinear Difference Equation to Study Highly Ductile Symmetric Fold (HDSF) Dynamics: a Conceptual Study
}

\author{
B.S. DAYA SAGAR* \\ Centre for Remote Sensing \& Information Systems, Department of Geoengineering, \\ Andhra University, Visakhapatnam 530 003, India
}

(Received 11 September 1998)

\begin{abstract}
Changes in morphology of a geological fold are due to stress and internally exerting forces (IEFs). Such morphological changes can be quantified in terms of fractal dimensions. Stress and the fractal dimension are depicted in normalized scale as dimensionless parameters. Incorporating these parameters in a first order nonlinear difference equation that has physical relevance as the simplest viable model of a symmetric fold sustaining morphological changes, numerical simulations are carried out which are analogous to creep experiments. In the first experiment, the constant stress $(\lambda)$ is employed to model the morphological dynamical behaviour of highly ductile symmetric folds (HDSFs) that are postulated as they are precarious to stress and IEF, and will not supervene the state of brittleness during the evolution. In the second experiment, the time dependent stress that is changed according to a dynamical rule is used to model distinct dynamical behaviors of these HDSFs. The results arrived through computer simulations are the attractor interlimb angles (AIAs). Bifurcation diagrams are also depicted to show the dynamical behaviors concerning the change in the stress dynamics.
\end{abstract}

Keywords: Stress dynamics, Symmetric fold, Normalized fractal dimension, Interlimb angles, Nonlinear

\section{INTRODUCTION}

The study of deformation in geological materials is one of the important tasks in structural geology. Fold one of such geological formations may be transformed due to mechanical properties. These transformations may be according to a rule through which one can predict the dynamical changes in folds. Several papers have emerged during the last decade, which cast the application of fractal concepts to study the fold mechanism. Several models are developed to study the folding processes and mechanisms [1-8]. Behavior of various systems of geoscientific interest such as electrical conductivity

* Fax: 0091891555547. 
and fractures of rocks to the microcrack population [9], coalescence of fractures [10,11] and stickslip behavior [12] through renormalization group approach, and the fault models using fractals and homogenization concepts [13] were studied. The rate of deformation depends not only on the rock mechanical properties of the geological formations and the energy acting on it but also on the antecedent morphological state of the fold. The shortening and amplification in the symmetric folds can be seen due to variations in the stress and ductility of the fold. Ductile folds are precarious to stresses. Moreover, fluctuations in the stress dynamics result in variations in the dynamical behavior of a symmetric fold ranging from steady state to periodicity and chaotic. The random behavior of fold, from its inception of the formation, is due to stress dynamics and the internally exerting forces that randomly influence the fold. The ductile folds of vertical axial type are subjected in the present qualitative investigation. The significant point is that this study is based on the posits that the deformation in the ductile fold is not permanent, and also that it will not ensue the state of brittleness during the influence of stress dynamics. In particular, the present paper deals with a continuous phase transition in a symmetric fold under dynamical conditions by considering the simple first order nonlinear difference equation. The logic behind using this equation in regard to understanding the fold morphological dynamics is follows

- The intensity of the cause can be derived from the effect. Such a derived cause might be in terms of various physical forces (stress \& IEF). The collectively acting coexisting physical forces is the cause to see its effect. This effect is in terms of deformation. Such a deformation can be quantified by means of an analytical value (e.g. fractal dimension [14]). By considering this quantified parameter at discrete time intervals, the term called stress regulatory force can be derived. These fractal dimensions at discrete time intervals enable that whether the dynamics of fold is of nonlinear type. However, based on the instinctive argument, it is apprehended that the fold dynamics follows nonlinear rules. This intutive argument may be endured by the fact that due to heterogeneous nature of external and internal stress influence, folds may undergo compression, amplification, cascade of compression-amplification and shear over a time interval. This argument is also supported by a postulate that the successive phases of a fold undergoing dynamics may be nonoverlapping; moreover, the output in terms of fractal dimension of the fold undergoing dynamics may not be directly proportional to its input. This phenomenon is due to the fact that the stresses and internally exerting forces are divergently balanced at discrete time intervals. These unequally balanced forces act against each other. Therefore, it is also visualized that the morphological dynamics of a fold is nonlinear. To carry out computer simulations to visualize distinct possible behaviors concerning a change in control parameter, a first order nonlinear difference equation (see (1)-(3)) proposed elsewhere [15], that has physical relevance as the simplest possible model of a HDSF undergoing morphological changes, is considered as the basis to further derive the Eqs. (4)-(7) and (11)-(14). Hence, qualitative studies have been carried out on understanding the fold morphological dynamics, and the acting stress dynamics of fold, by considering the first order nonlinear difference equation.

The organization of this paper is as follows. The definition of symmetric folds and the basic equations that are considered to study these symmetric folds are described in the Sections 2 and 3 respectively. In Sections 4 and 5, the procedure to compute the interlimb angles (IAs) of symmetric folds undergoing dynamics, and the relation between the normalized fractal dimensions and the IAs of these symmetric folds are respectively given. These sections are followed by the functional iteration to study the fold dynamical behavior and the computation of metric universality by considering the IAs 
in the Sections 6 and 7. In the end, the results of the computer simulations carried out on these symmetric folds, and the conclusions are briefly specified in the Sections 8 and 9 respectively.

\section{List of Symbols}

$N$ Number of limbs in a symmetric fold (3 for the fold type I and 2 for the fold type II)

$L$ Rigid length of the limb

$d$ Distance of the vertical projection of the upright symmetric fold

$D$ Fractal dimension $[\log N / \log (d / L)]$

$D_{T}$ Topological dimension, 0 , in 1-D space, 1 in 2-D space, and 2 in 3-D space.

$\alpha_{t}$ Normalized fractal dimensions (NFDs) at discrete time interval $(0<\alpha<1), \alpha=D-D_{\mathrm{T}}$

$\theta$ Interlimb angle (IA) $\left(\theta>60^{\circ}<180^{\circ}\right.$ for the symmetric fold type I; $\theta>90^{\circ}<180^{\circ}$ for the fold type II)

$\theta^{*}$ Attractor interlimb angle (AIA)

$\lambda$ Constant stress $(0<\lambda<1)$

$\lambda_{t}$ Time dependent stress parameter

$\mu$ Strength of stress modulation (SSM) parameter to compute time dependent stress parameter $(0<\mu<1)$

\section{DEFINITION OF SYMMETRIC FOLDS WITH 3 (FOLD TYPE I) AND 2 (FOLD TYPE II) LIMBS}

The description of the morphology of a fold pattern is mainly concerned with the outcrop of its profile. Generally, the nose of the fold is described as round or angular. If the limbs of a fold are of equal lengths, the fold is said to be symmetric (e.g. chevron or concordian fold) [16]. A typical asymmetric fold pattern is shown in Fig. 1(b) where one limb length differs with that of two other limbs. In this paper two types of upright symmetric folds of vertical axial type (e.g. zig-zig, chevron, or accordion folds) with rigid limbs (Fig. 1(a) and (c)) are considered. An upright symmetric fold (i.e. dip of the axial surface) with three limbs (Fig. 1(a)) with the following a)

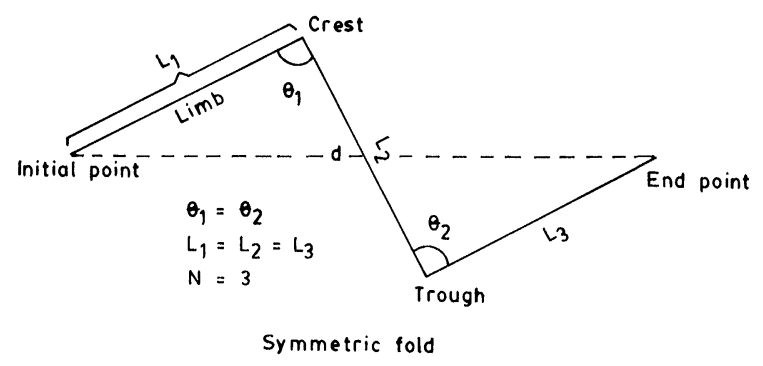

b)

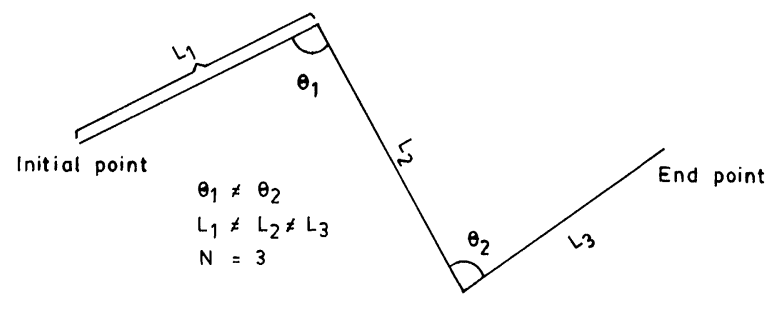

Asymmetric fold

c)

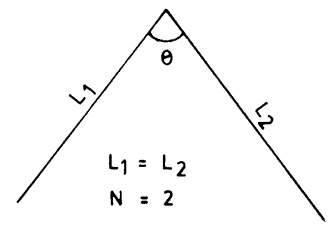

FIGURE 1 (a) Symmetric fold with three limbs, (b) a asymmetrical fold pattern and (C) symmetric fold with two limbs.

specifications is studied:

- Fold pattern should have three limbs $(N=3)$ with equal lengths $(L) ;\left(L_{1}=L_{2}=L_{3}\right)$, forming an anticline and a syncline.

- The angles $\left(\theta_{1}, \theta_{2}\right)$ between the two successive limbs should be equal $\left(\theta_{1}=\theta_{2}\right)$.

- The distance of vertical projection, $d$, should be greater than the length of a rigid $\operatorname{limb}(d>$ $\left.L_{1}=L_{2}=L_{3}\right)$.

An upright symmetric fold with two limbs (Fig. 1(c)) with the following specifications is also studied:

- Fold pattern should have two limbs $(N=2)$ with equal lengths $(L) ;\left(L_{1}=L_{2}\right)$ forming an anticline or a syncline.

- The distance of vertical projection, $d$, should be greater than the length of a rigid $\operatorname{limb}\left(d>L_{1}=L_{2}\right)$. 
TABLE I Four possible dynamics of symmetric fold

\begin{tabular}{|c|c|c|}
\hline Probable circumstances & Probable dynamical process & Trajectory behavior \\
\hline $\begin{array}{l}\text { A fold with high sinuosity index may } \\
\text { become straight }\end{array}$ & $\begin{array}{l}\text { Due to dominating internally acting } \\
\text { exerting force }\end{array}$ & Attracting to an initial conditions \\
\hline $\begin{array}{l}\text { A fold with medium sinuosity index may } \\
\text { increase as time progresses and then } \\
\text { converge to a point from which any } \\
\text { two patterns will overlap }\end{array}$ & $\begin{array}{l}\text { Due to unequal stress and the internally } \\
\text { acting force }\end{array}$ & Attracting to a fixed point \\
\hline $\begin{array}{l}\text { A fold oscillating between two } \\
\text { sinuosity indices }\end{array}$ & $\begin{array}{l}\text { Fold shape oscillating between two } \\
\text { points periodically. Shortening and } \\
\text { amplification, and vice versa }\end{array}$ & Oscillating between two points \\
\hline $\begin{array}{l}\text { A fold with either low or high tortuosity } \\
\text { may behave chaotically such that no two } \\
\text { patterns overlap }\end{array}$ & $\begin{array}{l}\text { Cascade of aperiodic stress and internally } \\
\text { exerting forces }\end{array}$ & Chaotically behaving \\
\hline
\end{tabular}

The length of the fold limb $(L)$ is considered as rigid when stress is acting on it. The stress concerned here is referred to horizontal stress only. Barring this, $d$ varies with the difference in the stress. The four possibilities of fold transformation that may arise in nature are presented Table I. If stress at discrete time intervals $\lambda_{t}>\lambda_{t+1}$ or $\lambda_{t+1}>\lambda_{t}$ play successively, the morphology of the HDSF changes which is obvious in geological context.

\section{BASIC EQUATIONS TO STUDY FOLD DYNAMICS}

The dynamical rule is visualized in the present investigation in two ways. They are according to the first order difference equation (2), and a modulated logistic equation (3). In the former case the stress regulatory parameter $\lambda$ is a constant stress control parameter, which acts against the internally exerting forces, whereas in the latter rule $\lambda_{t}$ is controlled by the strength of stress modulated parameter $\mu$ to understand the time dependent stress control parameter, $\lambda_{t}$, that describes the time dependent evolution of the fold morphological dynamics. Two types of fold dynamical systems are studied here:

(a) One that undergoes constant stress dynamics (CSD).

(b) One that undergoes time dependent stress dynamics (TDSD).

\section{First Order Difference Equation as a Dynamical Rule}

The fold morphological dynamics is controlled by a time dependent stress regulatory parameters.

The general from of the difference equation is taken as

$$
\alpha_{t+1}=f\left(\alpha_{t}\right)
$$

The dynamical behavior of symmetric fold under different total effective stresses is studied by following a function shown as a nonlinear first order difference equation (2). From the knowledge of the strain states of the fold at specific time intervals, the condition of the stress can be calculated. Force per unit area is stress. This is used to study the agents responsible for the deformation in the rock as it progressively changes shape. Such a study needs to investigate the nonlinear equations in which the stress that controls the fold dynamical system is constant during the evolution. To carry out such a study, Eq. (2) may be considered as a dynamical rule:

$$
\alpha_{t+1}=4 \lambda \alpha_{t}\left(1-\alpha_{t}\right)
$$

The limits of $\lambda$ are 0 and 1 , and the strains at respective states are quantified by $\alpha$ as 0 and 1 . The numerical representation 1 for $\lambda$, and $\alpha$ stand for any number, say $1000 \mathrm{kbar}$ and the upper limit of fractal dimension in normalized scale respectively. 


\section{Computation of SSM}

The SSM can be considered either as a constant or as time dependent parameter that controls the fold morphological dynamics. Rather than computing the physical forces that alter the fold dynamics, from the strain the dynamics of the stress regulatory parameter can be computed. The collective impact of such stresses (cause) which alter fold morphology can be defined by studying the (degree of deformation) effect due to the cause at discrete time intervals. As the fractal dimension enables the characteristic of the fold that is shortened as well as amplified, the parameter representing the strength of regulatory force can be defined as a numerical value. From the degree of deformation states at discrete time intervals, one can tell whether the stress influence is constant or not by fitting $\alpha_{t+1}$ vs $\alpha_{t}$ to fit the curve $\alpha_{t+1}=4 \lambda\left(1-\alpha_{t}\right)$. This derived stress is the slope value of the fitted curve. Such a value, $\lambda<1>0$, is considered as a constant stress. This constant stress also can be computed from the fractal dimensions of a fold at discrete time intervals. The fluctuations in the fold morphology depend on both the changes in the stress intensity and in the original constitution of the fold. If one knows the stress states at different time intervals, say $\lambda, \lambda_{t+1}, \ldots, \lambda_{t+n}$, the $\operatorname{SSM}(\mu)$ can be derived to compute the time dependent stress states by plotting $\lambda_{t+1}$ vs $\lambda_{t}$ to fit the curve $\lambda_{t+1}=4 \mu\left(1-\lambda_{t}\right)$ : It is hypothesized as the time dependent stress regulatory parameter attains higher value, at the subsequent times it is controlled by the factor $\left(1-\lambda_{t}\right)$. It is visualized that if the stress regulatory force is high, make it small, and vice versa. This is a wonderful recipe to carry out simulation numerically. The time dependent stress that, in turn, controls the fold morphology can be computed from the stress states in a time series form. This aspect is to study the coupled systems. In this coupled system, which is detailed in the sequel, the stress and fold morphological dynamics are interdependent.

\section{Symmetric Fold Dynamics Under the Influence of Constant Stress}

A fold with high sinuosity will have an interlimb angle (IA) of $\theta=60^{\circ}$ (for 3-limb fold) and $90^{\circ}$ (for 2-limb fold), and for a linear fold, $\theta=180^{\circ}$. A fold with high sinuosity will have a value of $\alpha$ approaching 1 , and for a straight line, $\alpha=0$. The upper and lower limits of $\alpha$, viz. 0 and 1 , arise at lowest and greatest stress states viz. $\lambda=0$ and 1 respectively. The parameter $\lambda$ gives total description of the dynamics of fold. The impact of the unequal compressive forces on a symmetric fold in terms of its dynamical behavior is investigated through the first order difference equation of the form $\alpha_{t+1}=f\left(\alpha_{t}\right)$; the fractal dimension in normalized scale at $t+1, \alpha_{t+1}$, is given as some function, $f$, of the fractal dimension at time $t, \alpha_{t}$. If this equation were linear $(f=\lambda \alpha)$, the fractal dimension would simply increase or decrease exponentially if $\lambda<0.25$. Moreover, the fractal dimension tends to increase when at low $\alpha$ and to crash at high $\alpha$ value, corresponding to some nonlinear function, with a hump, of which the quadratic is $f=\alpha_{t+1}=\lambda \alpha(1-\alpha)$. It does mean that there is a tendency for the variable $\alpha$ to increase from time " $t$ " to the next when it is small, and for it to decrease when it is large. When the symmetric fold possesses less fractal dimension, there may be a possibility for it to get compressed due to stresses that dominate internal force. When it possesses high fractal dimension, due to internal forces that dominate the stress acting against, this may lead to a decline of the fractal dimension. This tendency is due to the fact that the internally exerting forces dominate the impact of stresses. The impacts of internal forces fluctuate. These fluctuating impacts depend on the $\alpha$ values. The reason behind this possibility may be the fact that during the fold dynamics, unequal internal forces influence fold at discrete time intervals, and also the variations in the strength of the fold itself. This tendency is preserved due to $\left(1-\alpha_{t}\right)$ in Eq. (2). Equation (2), to compute $\alpha_{t+1}$, $\lambda \alpha_{t}\left(1-\alpha_{t}\right)$, explains that the normalized status of a symmetric fold dynamics if $\alpha$ starting at larger than 1 , it immediately goes negative at one time step. Moreover, if $\lambda>1$, the hump of the parabola exceeds 1 , thus enabling the initial $\alpha$ value near 0.5 to shear in two time steps. Therefore, the analysis is restricted to value of $\lambda, \alpha$ between 0 and 1 . It is also interesting to study the critical states from which the 
internal forces dominate the external stresses (CSD). The impacts of such internal forces acting alternatively are predominant at larger threshold regulatory stresses. This idea can be seen from the depicted bifurcation diagrams in Section 8 .

In qualitative understanding of the dynamical behavior, the value $\alpha_{t+1}$ is obtained from the previous value $\alpha_{t}$ by multiplying it by $4 \lambda\left(1-\alpha_{t}\right)$, it is clear that for $4 \lambda\left(1-\alpha_{t}\right)$ greater than 1 , the successive values, viz, $\alpha_{t+2}, \alpha_{t+3}, \alpha_{t+4}, \ldots, \alpha_{t+N}$, will grow bigger - that is, a change in $\alpha_{t}$ will get amplified. This is the fold shortening due to relatively high stress. However, $\alpha_{t}$ cannot increase indefinitely because of the mechanical properties of the geological material make up of the stratum. $4 \lambda\left(1-\alpha_{t}\right)$ becomes smaller than 1 , and the subsequent values must diminish. In the context of fold dynamics, this is fold stretching (amplification) due to high impact of exerting forces that dominate the stress. To determine the stability concerning incessantly acting stress with different magnitudes, a linearized analysis may be conducted through the studies of the dynamical behaviors of a model that is described by the first order difference equation, which consist in finding constant equilibrium solutions.

\section{Fold Morphological Dynamics Under the Influence of Time Dependent Stress}

In contrast to the fold dynamics, under the influence of constant stress, the behavior variations may be observed when stress is made time dependent. This idea is induced from the following statement of Ruelle [17]. It states that the behavior of a dynamical system can be studied with adiabatically fluctuating parameters where the control parameter has a very slow variation in time and this time dependence itself might be determined by a dynamics. This is the origin to consider the stress as a time dependent parameter that controls the fold morphological dynamics. Besides this, the logic behind using the time dependent stress dynamics (TDSD) is that the complexity of fold morphological dynamics depends on the complexity of stress dynamics. Hence, in under- standing the fold dynamics, the dynamics of the stress should also be understood.

\section{The Dynamics of the Time Dependent Stress}

A possibility for stress being a time dependent parameter, which may be confirmed from the fact that the stress influence is not homogeneous in the time domain. In such a case understanding the dynamics of stress is an important event. However, we assumed that the stress at time $t+1$ is not directly proportional to the stress at time $t$. This engendered to consider the first order nonlinear difference equation as a rule to understand the stress dynamics also (Eq. (3)). In (Eq. (3)), $\lambda_{t}$ is a time dependent stress and $\mu$ is the strength of stress modulated parameter (SSMP) that controls the time dependent stress dynamics. By considering this time dependent stress $\left(\lambda_{t}\right)$, the degree of deformation at discrete time intervals may be studied by the modulated logistic equation (3). To show the effect of time dependent stress regulatory parameter on the fold dynamical system, Eq. (3) is considered to carry out numerical simulation. For better understanding, see return maps for the TDSD in Fig. 5.

In Eq. (3), the behavior of $\alpha$ is controlled by the behavior of $\lambda$. This is explored as the fold and the stress, that is represented in numerical form $\lambda<1>0$, dynamical systems, in which the behavior of the fold morphology depends on the behavior of $\lambda$. It means this coupled system contains two dynamical systems, in which the dynamical parameters are $\alpha$ and $\lambda_{\tau}$. The equation to describe this coupled system is written from Eq. (2) as

$$
\alpha_{t+1}=4 \lambda_{t} \alpha_{t}\left(1-\alpha_{t}\right), \quad \lambda_{t+1}=4 \mu \lambda_{t}\left(1-\lambda_{t}\right) .
$$

Various phases of fold dynamics, under the influence of constant and time dependent stresses, that can undergo can be studied by following Eqs. (2) and (3) respectively. In Eqs. (2) and (3), a detailed form of forces and fluxes will be indirectly represented by $\lambda$ (constant stress dynamics) or $\mu$ (strength of stress modulation to model the time dependent stress). 


\section{COMPUTATION OF IA ( $\theta)$ OF CORRESPONDING NFD $(\alpha)$ OF A SYMMETRIC FOLD UNDER DYNAMICS}

By considering the parameters such as fractal dimension (Mandelbrot, 1982), in normalized scale $\alpha$ to describe the change in morphology of the fold, and the constant $(\lambda)$ and the time dependent stress regulatory parameter $\left(\lambda_{t}\right)$ to describe the detailed form of forces and fluxes in the proposed equations (4)-(7) and (11)-(14), the dynamical behavior of symmetric fold types I and II that may behave from stable to chaotic can be quantified.

Fold type I Equations (4) and (5) are proposed which include certain specifications of a symmetric fold type I under evolution according to Eq. (2) to record the changing IAs $(\theta)$ for both constant (Eq. (4)) and time dependent (Eq. (5)) stress regulatory parameters:

$$
\begin{aligned}
& \theta_{t+1}=\cos ^{-1} \frac{5-10^{\left\{2 \log N /\left[4 \lambda \alpha_{t}\left(1-\alpha_{t}\right)+D_{\mathrm{T}}\right]\right\}}}{4}, \\
& \theta_{t+1}=\cos ^{-1} \frac{5-10^{\left\{2 \log N /\left[4 \lambda_{t} \alpha_{t}\left(1-\alpha_{t}\right)+D_{\mathrm{T}}\right]\right\}}}{4} .
\end{aligned}
$$

Fold type II Equations (6) and (7) are proposed to compute the IA for the symmetric fold type II which is under evolution according to a rule of Eq. (2). These equations, (6) and (7), are proposed respectively for both constant $(\lambda)$ and time dependent $\left(\lambda_{t}\right)$ stress regulatory parameters:

$$
\begin{aligned}
& \theta_{t+1}=2 \sin ^{-1} \frac{10^{\left\{\log N /\left[4 \lambda \alpha_{t}\left(1-\alpha_{t}\right)+D_{\mathrm{T}}\right]\right\}}}{2}, \\
& \theta_{t+1}=2 \sin ^{-1} \frac{10^{\left\{\log N /\left[4 \lambda_{t} \alpha_{t}\left(1-\alpha_{t}\right)+D_{\mathrm{T}}\right]\right\}}}{2} .
\end{aligned}
$$

\section{THE RELATION BETWEEN $\alpha$ AND $\theta$}

The $\alpha$ and $\theta$ are respectively denoted for the fractal dimension in normalized scale and the IA of the symmetric fold. As the fold is contracted, horizontally in such a way that the limbs $(L)$ will not change and by having the change in $d$, the IAs $(\theta)$ will be changed. A symmetric fold with high degree of linearity (straight) approximately possesses $180^{\circ}$ IA. A fold with high sinuosity such that it is selfavoiding at any higher magnifications possesses $60^{\circ}$. A symmetric fold with $60^{\circ}$ and $180^{\circ}$ of IAs possesses fractal dimensions 2 and 1, respectively. However, these two limits of IAs for the type II fold are respectively $90^{\circ}$ and $180^{\circ}$. A symmetric fold under dynamics will reach to criticality where the ratio between $\log (N)$ and $\log (d / L)$ becomes 2 . At this critical state, the inter-limb angle becomes $60^{\circ}$ that is called critical angle, $\theta_{\text {crit }}$. This critical angle for the symmetric fold type II is $90^{\circ}$. A symmetric fold under study is self-avoiding if and only if, $\theta>\theta_{\text {crit }}$. With $\theta<\theta_{\text {crit }}$ fold pattern gets sheared. At the critical angle, $\theta_{\text {crit }}$, the parameter $\alpha$ attains its peak value, $\alpha=1$. The corresponding fractal dimension is at its criticality, i.e., $\alpha+D_{\mathrm{T}}=2$, for intersecting. With $\alpha_{t}$ and $\lambda$ as 0.5 and 1 respectively, the $\alpha$ value of fold under evolution at time $t+1$ becomes 1 at one single time step, and the $\theta$ will be found at its criticality. Once the inter-limb angle reaches its criticality, the symmetric fold may become either stable, or stretched, or breaks as the influence of the stress continues. Equations (4)-(7) and (11)-(14) are to observe how the IAs are restricted between $180^{\circ}$ and $60^{\circ}$, and $180^{\circ}$ and $90^{\circ}$ for the fold types I and II respectively under the influence of CSD and TDSD. The latter values, $60^{\circ}$ and $90^{\circ}$, are critical angles beyond which the folds self-intersect. The magnitude of variation in the $\theta$ 's from time $t$ to $t+1$ depends on the intensity of the stress and the internally exerting forces that the fold is subjected to. As shown in Eq. (2), $\alpha \in[0,1]$ representing the fold with linearity and with the greatest possible contortion respectively. The corresponding $\theta$ 's at the $\alpha=0$ and 1 are computed as $180^{\circ}$ (lower limit) and $60^{\circ}$ (upper limit), and $180^{\circ}$ (lower limit) and $90^{\circ}$ (upper limit) for symmetric folds with three and two limbs respectively. It is worth mentioning that the fold, possessing parasitic folds, will self-intersect at less than the upper limits viz $60^{\circ}$ and $90^{\circ}$ for the two type of folds. The lower and upper limits represent the most probable contorted fold at which the parasitic folds will self-intersect, and linear structure 
before getting to be folded respectively. It is essential to mention that the first order fold at various magnifications contains parasitic folds that contain still minor folds, and so on. Up to $60^{\circ}$ of IA of a symmetric fold at any higher magnification, minor folds that possess exact self-similarity will not self-intersect. With the IA of a first order symmetric fold with lesser than the critical angle, minor folds will self-intersect. For better comprehension, this phenomenon is represented diagrammatically in Fig. 2. From $\theta$, the IA, the corresponding NFD can be calculated for the symmetric folds with three and two limbs respectively from Eqs. (8) and (9):

$\alpha=2 \log N /[\log (5-4 \cos \theta)]-D_{\mathrm{T}} \quad($ for $N=3)$,

$\alpha=\log N /\left[\log [2 \sin (\theta / 2)]-D_{\mathrm{T}} \quad(\right.$ for $N=2)$.

The above expressions give the NFD of the symmetric folds with three limbs and two limbs. The corresponding NFDs for these folds with $\theta>60^{\circ}, 90^{\circ}<180^{\circ}$ are $\alpha<1>0$.

(a)

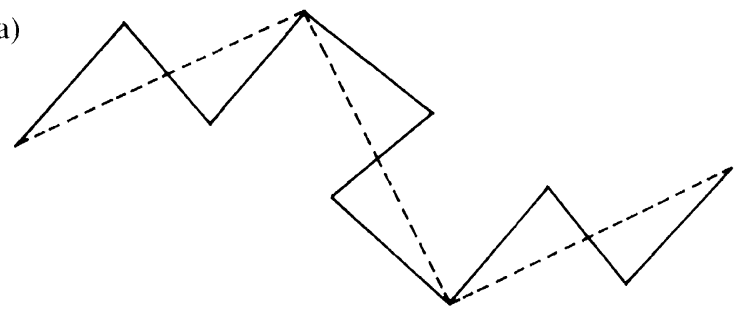

(b)

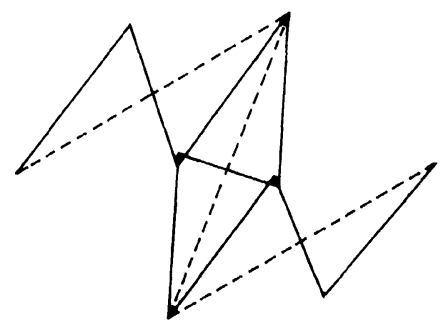

FIGURE 2 Symmetric folds with several folds of different IAs are shown schematically. (a) Schematic of self-avoiding symmetric fold profile with second order folds. The IA of first order fold (shown in dotted line) is greater than $60^{\circ}$ and (b) a schematic of self-intersecting symmetric fold profile with second order folds. The IA of first order fold (shown as dotted line) is lesser than critical angle, i.e., $60^{\circ}$. Hence, it is selfintersecting. The intersecting second order folds may be seen.

\section{FUNCTIONAL ITERATION BY CONSIDERING $\theta$ 'S AT DISCRETE TIME INTERVALS}

Instead of considering the $\alpha$ 's one can consider the $\theta$ values to carry out simulations for fold modeling. Equations (11)-(14) are proposed in which the IAs are considered instead of the NFDs to compute the IAs of the fold undergoing dynamics according to the first order difference equation as a dynamical rule. These equations are similar to the Eqs. (4)-(7).

It is intended to compute the IAs at time $t+1$ by considering $\theta$ at time $t$ as some function from the relation between $\alpha$ and $\theta$ described in Section 5. The following generalized equation which is akin to that of Eq. (1) is considered to perform functional iteration:

$$
\theta_{t+1}=f\left(\theta_{t}\right)
$$

The function in Eq. (10) is expanded as follows by substituting Eqs. (4) and (8) for the fold with three limbs that is undergoing dynamics as

$$
\begin{aligned}
& \theta_{t+1} \\
& \qquad \cos ^{-1}([5-10 \exp 2 \log N \\
& /\left\{4 \lambda\left\{2 \log N /[\log (5-4 \cos \theta)]-D_{\mathrm{T}}\right\}\right. \\
& \times\{1-\{2 \log N /[\log (5-4 \cos \theta)] \\
&\left.\left.\left.\left.\left.-D_{\mathrm{T}}\right\}\right\}+D_{\mathrm{T}}\right\}\right] / 4\right) .
\end{aligned}
$$

The expression as an exponent is based on the first order nonlinear difference equation. In the above equation, the strength of stress regulatory force is a constant stress regulatory parameter. However, the emphasis is also given in the present investigation to carry out the iterations to understand the possible dynamics by understanding the dynamics of the time dependent stress regulatory parameter. This function for the time dependent stress regulatory parameter is defined in which 
Eqs. (5) and (8) are considered:

$$
\begin{aligned}
\theta_{t+1} & \\
=\cos ^{-1} & \left(\left[5-10 \exp 2 \log N /\left\{4 \lambda_{t}\{2 \log N\right.\right.\right. \\
& \left./[\log (5-4 \cos \theta)]-D_{\mathrm{T}}\right\}\{1-\{2 \log N \\
& \left.\left.\left.\left.\left./[\log (5-4 \cos \theta)]-D_{\mathrm{T}}\right\}\right\}+D_{\mathrm{T}}\right\}\right] / 4\right) .
\end{aligned}
$$

The function expressed in the Eq. (10) is expanded as Eq. (13) by considering Eq. (6) and (9) as follows for the symmetric fold with two limbs:

$$
\begin{aligned}
\theta_{t+1} & \\
=2 \sin ^{-1} & ([10 \exp \log N /\{4 \lambda\{\log N \\
& \left./[\log [2 \sin (\theta / 2)]]-D_{\mathrm{T}}\right\} \\
& \times\{1-\{\log N /[\log [2 \sin (\theta / 2)]] \\
& \left.\left.\left.\left.\left.-D_{\mathrm{T}}\right\}\right\}+D_{\mathrm{T}}\right\}\right] / 2\right) .
\end{aligned}
$$

By substituting the time dependent stress regulatory parameter $\left(\lambda_{t}\right)$ the Eq. (13) is rewritten follows:

$\theta_{t+1}$

$$
\begin{aligned}
=2 \sin ^{-1} & \left(\left[10 \exp \log N /\left\{4 \lambda_{t}\{\log N\right.\right.\right. \\
& \left./[\log [2 \sin (\theta / 2)]]-D_{\mathrm{T}}\right\} \\
& \times\{1-\{\log N /[\log [2 \sin (\theta / 2)]] \\
& \left.\left.\left.\left.\left.-D_{\mathrm{T}}\right\}\right\}-D_{\mathrm{T}}\right\}\right] / 2\right) .
\end{aligned}
$$

Symmetric fold dynamical behaviors can be studied by these equations.

\section{COMPUTATION OF METRIC UNIVERSALITY BY CONSIDERING THE AIAS OF SYMMETRIC FOLDS UNDER DYNAMICS}

The critical states are broadly categorized as

- attracting to initial state,

- attracting to a fixed point state,

- oscillating between two points - period 2,

- period 3 and chaotic state of fold dynamics.
The threshold stress regulatory parameter is the value at which the symmetric fold under dynamics produces critical state(s) or attractor(s). These threshold stress regulatory parameter, for CSD $(\lambda)$, and $\operatorname{TDSD}(\mu), \lambda_{1}, \mu_{1}=0.75 ; \lambda_{2}, \mu_{2}=0.865$; $\lambda_{3}, \mu_{3}=0.89225$; and $\lambda_{4}, \mu_{4}=0.8925$. The parameters $\lambda$ and $\mu$ respectively represent the constant and SSM to simulate time dependent stress regulatory parameters considered for fold dynamical systems respectively. Feignbaum [18] proposed the universality constant, i.e., $4669 \ldots$ for the celebrated nonlinear first order difference equation (2). Similarly, the distance between the openings of attractors at respective threshold stress regulatory parameters are considered to compute metric university $(\delta)$, that converges to 2.5069 [18]. The AIAs are computed (Table I(a) and (b)) for both the types of fold systems that are controlled by both constant and time dependent stress regulatory parameters. By considering these AIAs of coupled and non-coupled fold dynamical systems, Eqs. (15) and (16) to compute Feignbaum's metric universality constant $(\delta)$ for both the types of fold morphological dynamics are proposed.

Fold type $I$ The parameter $\delta$ that converges to 2.5069 can be computed for the symmetric fold under dynamics by considering the AIAs by Eq. (15):

$$
\begin{aligned}
\delta \sim & \left(\left\{\log \left(5-4 \cos \theta_{N+1}^{*}\right)\right\}-\left\{\log \left(5-4 \cos \theta_{N}^{*}\right)\right\}\right. \\
& \left.\times\left\{\log \left(5-4 \cos \theta_{2 N+2}^{*}\right)\right\}\left\{\log \left(5-4 \cos \theta_{2 N+3}^{*}\right)\right\}\right) \\
& /\left(\left\{\log \left(5-4 \cos \theta_{N}^{*}\right)\right\}\left\{\log \left(5-4 \cos \theta_{N+1}^{*}\right)\right\}\right. \\
& \left.\times\left\{\log \left(5-4 \cos \theta_{2 N+3}^{*}\right)\right\}-\left\{\log \left(5-4 \cos \theta_{2 N+2}^{*}\right)\right\}\right),
\end{aligned}
$$

where $N=2,4,8,16, \ldots$

Fold type II AIAs are liable to vary with the type of fold. The parameter $\delta$ can be computed for the symmetric fold type II under dynamics by considering the AIAs by Eq. (16). For this type of fold, the attractor IAs for the two dynamical rules 
will be computed according to Eqs. (13) and (14):

$$
\begin{aligned}
\delta \sim & \left(\left\{\log \left[2 \sin \left(\theta_{N+1}^{*} / 2\right)\right]-\log \left[2 \sin \left(\theta_{N}^{*} / 2\right)\right]\right\}\right. \\
& \left.\times \log \left[2 \sin \left(\theta_{2 N+2}^{*} / 2\right)\right] \log \left[2 \sin \left(\theta_{2 N+3}^{*} / 2\right)\right]\right) \\
& /\left(\log \left[2 \sin \left(\theta_{N}^{*} / 2\right)\right] \log \left[2 \sin \left(\theta_{N+1}^{*} / 2\right)\right]\right. \\
& \left.\times\left\{\log \left[2 \sin \left(\theta_{2 N+3}^{*} / 2\right)\right]-\log \left[2 \sin \left(\theta_{2 N+2}^{*} / 2\right)\right]\right\}\right),
\end{aligned}
$$

where $N=2,4,8,16, \ldots$

\section{RESULTS OF SIMULATIONS}

The recent advancement is that the nonlinear differential equations are used to represent the motion of the actual processes in the form of "maps". Several natural phenomena of geoscientific interest are modeled. The cogency of the model can be justified provided the large amount of time series data is procurable. Such time series data enable one to find whether the attractor that describes the evolutionary pattern of the folds possesses low dimensionality. However, in the present section, the time series data that reveal the possible dynamics of the stress and the fold morphology is simulated to show the qualitative characteristics. Two cases have been considered, of which the first one is by following the constant stress dynamics and, in the second one, the time dependent stress dynamics is followed.

\section{Fold Dynamical System Under the Influence of Constant Stress}

A case study is shown by considering the symmetric fold type I for better understanding. By changing $\lambda$, the constant stress, with a fixed initial $\alpha$ value, two possible states of dynamical behaviors are simulated qualitatively and illustrated Fig. 3(a) and (b). Based on Eq. (4) two sets of conditions are considered to transform a symmetric fold qualitatively with $\alpha_{t}$ as 0.0636314 and control parameter $\lambda$ as 0.975 (chaotic (a)

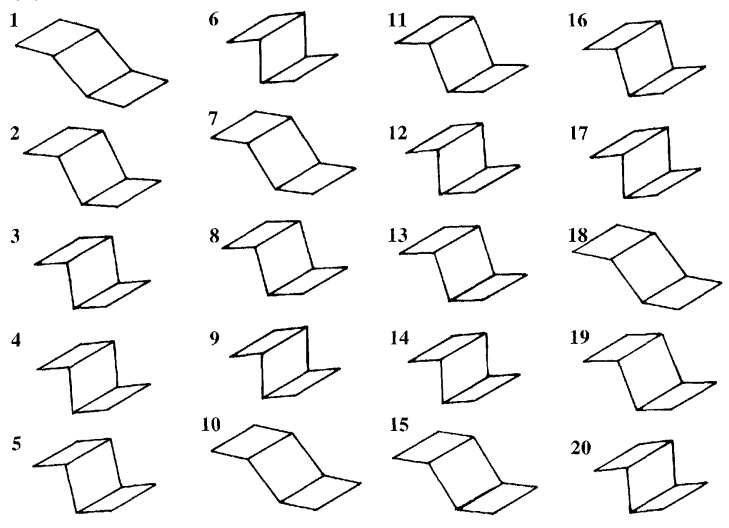

(b)

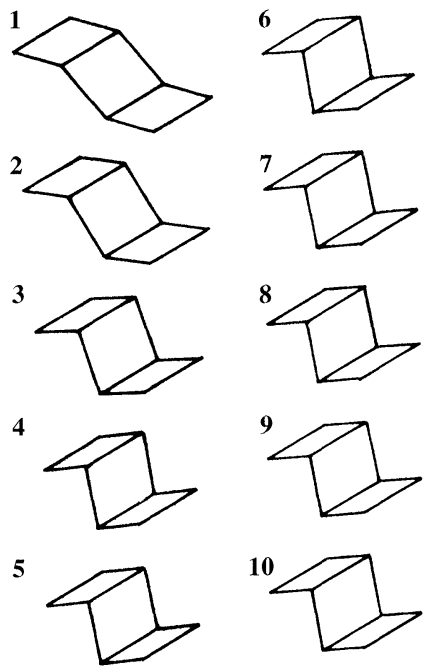

FIGURE 3 Evolution of a fold type I with strength of nonlinearities (a) $\lambda=0.975$ and (b) $\lambda=0.7$. The numbers represent the discrete times.

attractor) and 0.7 (fixed point attractor). The IAs $(\theta)$ of dynamically changing symmetric fold are computed by Eq. (4) and the parameters of the symmetric fold under study are presented in Table III. Figures 3(a) and 3(b) show simulated fold at successive stages of evolution under different constant stress control parameters represented as $\lambda$. To illustrate the chaotic fluctuations in the symmetric fold evolution, with $\lambda=0.975$ the evolution process is simulated on computer (Fig. 3(a)). During this evolution progressive compressions are followed by amplification randomly. In Fig. 3(a), the fold was 
TABLE II Certain essential parameters of the fold behavior model

\begin{tabular}{|c|c|c|c|c|}
\hline$t$ & $\alpha$ & $d$ & $\%$ of shortenings with respect to initial fold & $\theta$ (deg.) \\
\hline & \multicolumn{4}{|c|}{$L=2.1364248 ; \lambda=0.975$} \\
\hline 1 & 0.0636314 & 6 & 0 & 136.20495 \\
\hline 2 & 0.2323667 & 5.2101002 & 13.17 & 103.69863 \\
\hline 3 & 0.6956524 & 4.0838214 & 31.94 & 70.334921 \\
\hline 4 & 0.8257085 & 3.8996213 & 35.01 & 65.350395 \\
\hline 5 & 0.5612645 & 4.3180416 & 28.04 & 76.777388 \\
\hline 6 & 0.960362 & 3.7417248 & 37.64 & 61.108436 \\
\hline 7 & 0.1484606 & 5.5607296 & 7.3212 & 116.33816 \\
\hline 8 & 0.4930383 & 4.4591445 & 25.681 & 80.740878 \\
\hline 9 & 0.974811 & 3.7264139 & 37.893 & 60.697772 \\
\hline 10 & 0.0957626 & 5.8225257 & 2.958 & 127.36545 \\
\hline 11 & 0.3377092 & 4.8568865 & 19.06 & 92.41023 \\
\hline 12 & 0.8722808 & 3.8416856 & 35.99 & 63.791861 \\
\hline 13 & 0.4344873 & 4.5951013 & 23.42 & 84.636437 \\
\hline 14 & 0.9582616 & 3.7439746 & 37.6 & 61.168786 \\
\hline 15 & 0.1559856 & 5.5262096 & 7.9 & 115.00552 \\
\hline 16 & 0.511379 & 4.419505 & 26.34159 & 79.587900 \\
\hline 17 & 0.974495 & 3.726746 & 37.88757 & 60.682251 \\
\hline 18 & 0.096933 & 5.816304 & 3.0616 & 127.028912 \\
\hline 19 & 0.341393 & 4.845945 & 19.23425 & 92.039776 \\
\hline 20 & 0.876891 & 3.836153 & 36.06412 & 63.617582 \\
\hline \multicolumn{5}{|c|}{$L=2.1364248 ; \lambda=0.7$} \\
\hline 1 & 0.0636314 & 6 & 0 & 136.20495 \\
\hline 2 & 0.1668308 & 5.4776101 & 8.706498 & 113.1671 \\
\hline 3 & 0.3891952 & 4.7112823 & 21.478628 & 88.037037 \\
\hline 4 & 0.6656225 & 4.1318048 & 31.136587 & 71.643445 \\
\hline 5 & 0.6262268 & 4.198355 & 30.027417 & 73.46716 \\
\hline 6 & 0.655387 & 4.1486897 & 30.85517 & 72.105131 \\
\hline 7 & 0.6322937 & 4.1876539 & 30.205768 & 73.173163 \\
\hline 8 & 0.6509213 & 4.1561442 & 30.73093 & 72.309177 \\
\hline 9 & 0.635064 & 4.183054 & 30.282433 & 73.017503 \\
\hline 10 & 0.648922 & 4.159500 & 30.675 & 72.371934 \\
\hline
\end{tabular}

progressively compressed, which is due to horizontal stress upto discrete time, $t=6$. The fold at discrete time $t=6$ (approaching critical angle, $\theta=61^{\circ}$ ) gets amplified due to dominating internal forces at time $t=7$. At discrete times $t=7,10,15,18$ the fold amplification in the fold profile can be seen due to higher internal forces than the CSD parameter. These observations can be seen from the numerically represented parameters depicted in Table II. This fold evolution process is represented qualitatively through graphic analysis. It represents the qualified dynamical behavior of the evolving fold in a quantitative manner. Figure 4(a) shows the return map, in which chaotic behavior of the trajectory can be seen. In Fig. 3(b), symmetric fold was compressed progressively. The compression is due to horizontal stress. It may be observed that after discrete time $t=5$, the fold has reached equilibrium state. This evolution is also qualitatively represented through graphic analysis in Fig. 4(b) in which the trajectory is attracting to a fixed point. Instead of the fractal dimensions in normalized scale, their corresponding IAs are represented on return maps. It is observed when the $\alpha$ values lie between 0 and 1 , their corresponding interlimb angles will be between $180^{\circ}$ and $60^{\circ}$, respectively. AIAs at respective threshold stress regulatory parameters are computed (Table III) for the fold type I under dynamics by considering the initial fold specification with $\alpha=0.00001 \quad\left(\theta=179.43028^{\circ}\right)$. The number of iterations performed are $3 \times 10^{4}$ time steps. 
(a)

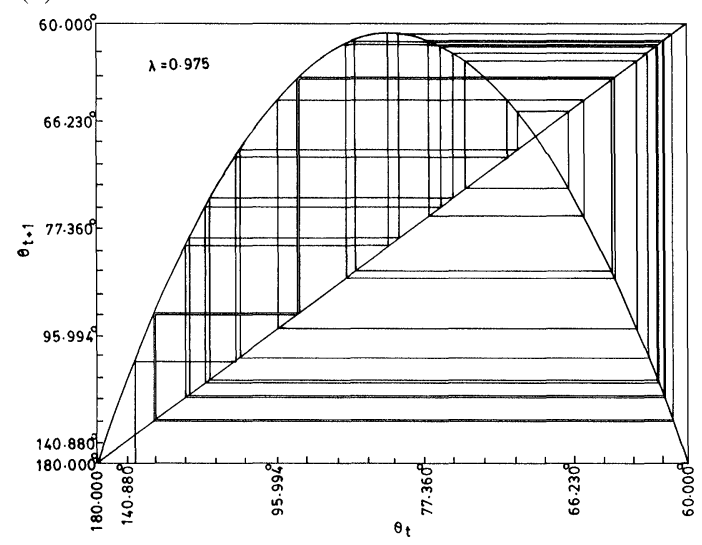

(b)

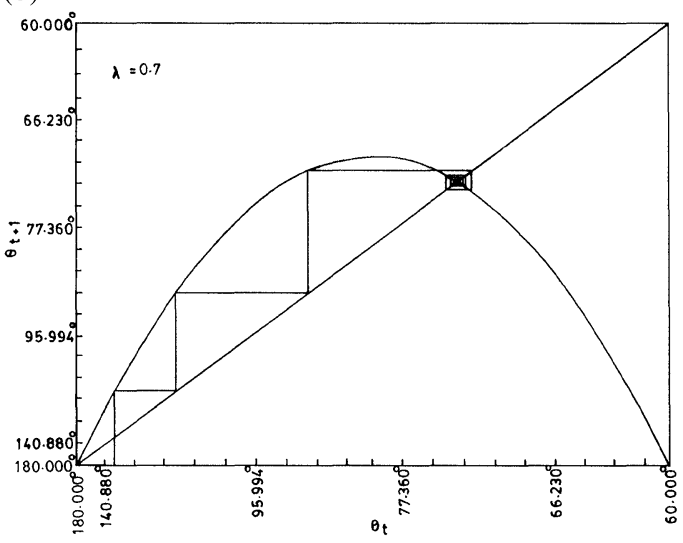

FIGURE 4 Logistic maps for the qualitative dynamical behavior of symmetric folds under evolution shown in (a) and (b). It may be seen that the values mentioned on the abscissa are IAs in degrees for the symmetric fold with three limbs.

\section{Fold Dynamical System Under the Influence of Time Dependent Stress}

It is assumed that the fold dynamical system is controlled by the TDSD. Hence, the study of fold dynamics system is treated as a coupled system. The stress dynamics is simulated by considering the first order nonlinear difference equation as the basis to further generate the time dependent stress regulatory parameter. With this simulated time dependent stress regulatory parameter the fold dynamics is controlled. With different possible TDSD, the symmetric fold dynamics is studied, and sets of equations are proposed in which the dynamically changing parameters are IAs and the AIAs. Some interesting results have been arrived at when the stress regulatory parameter is made time dependent. At the threshold stress regulatory parameter in the coupled system, i.e., $\mu$, the time dependent stress regulatory parameter $\lambda_{t}$, the attractor NFDs and the corresponding AIAs are computed and compared with the results for the autonomous fold dynamical system which is controlled by the non-time-dependent stress regulatory parameter.

Return maps are plotted for the low dimensional deterministic randomness of the dynamical system of time dependent stress regulatory parameter $\left(\lambda_{t+1}-\lambda_{t}\right.$ vs $\left.\lambda_{t+2}-\lambda_{t+1}\right)$ (Fig. 5(a) - (c)), and the fold morphology dynamical system that is controlled by the time dependent stress regulatory parameter (i.e $\theta_{t+1}-\theta_{t}$ vs $\theta_{t+2}-\theta_{t+1}$ ) (Fig. 6(a)(c)). Plots are constructed by considering the differences of successive $\theta$ values in the time domain $t$ in $\theta_{t}$ and $\theta_{t+I}$ phase space (Fig. 6(a) $-(\mathrm{c})$ ). These return maps indicate the characteristic behavior of the simulated time dependent stress and fold dynamical systems. This demonstrates that one can analyze the temporal aspects of a system in the same manner as used to analyze time series data of a system variable. These return maps are plotted by considering the variables $\lambda_{t}=0.00001 ; \mu=0.9,0.95$, $0.9925 ; \alpha_{t}=0.00001$ or $\theta_{t}=179.43028$; no. of iterations $=10 \times 10^{6}$ time steps. The AIAs are also computed by iterating Eqs. (11), (13) and (12), (14) respectively for the two symmetric fold dynamical systems under the influence of constant and time dependent stresses Table III and IV. The difference in the AIAs from the type I to type II symmetric folds is apparent. The variation is also observed in the AIAs in these two types of folds when they are subjected to the dynamical rules that include TDSD and CSD (Table IV). These AIAs are liable to vary with the variations in the fold specifications, dynamical rules involved in the fold morphological dynamics and in the stress dynamics. For instance, if the rule that controls the stress dynamics is a linear equation, contrary to the dynamical rule considered in this study, the AIAs are liable to vary. This important point can be further justified by 
TABLE III AIAs at the threshold regulatory forces after $3 \times 10^{4}$ time steps: The dynamical rule is simple first order nonlinear difference equation with constant stress control parameter

\begin{tabular}{|c|c|c|c|c|c|c|c|c|c|}
\hline \multicolumn{2}{|c|}{$\begin{array}{l}\text { Threshold control } \\
\text { parameter }\end{array}$} & \multicolumn{8}{|c|}{ AIAs (deg.) } \\
\hline \multicolumn{10}{|c|}{ Symmetric fold type $\mathrm{I}\left(\alpha=0.00001, \theta_{\text {init }}=179.43028^{\circ}\right)$} \\
\hline \multicolumn{10}{|c|}{$\begin{array}{lll}\lambda_{1} & 0.75 & \theta_{1}=71.663691\end{array}$} \\
\hline$\lambda_{2}$ & 0.865 & \multicolumn{2}{|c|}{$\theta_{3}=64.896443$} & \multicolumn{2}{|c|}{$\theta_{4}=86.185576$} & \multicolumn{2}{|c|}{$\theta_{5}=64.14859$} & \multicolumn{2}{|c|}{$\theta_{6}=82.378284$} \\
\hline$\lambda_{3}$ & 0.89225 & \multicolumn{2}{|c|}{$\theta_{7}=63.154877$} & \multicolumn{2}{|c|}{$\theta_{8}=80.241149$} & \multicolumn{2}{|c|}{$\theta_{9}=65.166519$} & \multicolumn{2}{|c|}{$\theta_{10}=89.678203$} \\
\hline & & \multicolumn{2}{|c|}{$\theta_{11}=63.451428$} & \multicolumn{2}{|c|}{$\theta_{12}=77.326159$} & \multicolumn{2}{|c|}{$\theta_{13}=65.932067$} & \multicolumn{2}{|c|}{$\theta_{14}=91.542719$} \\
\hline \multirow[t]{2}{*}{$\lambda_{4}$} & 0.8925 & 63.15 & 80.533198 & 65.095265 & 89.514049 & 63.473466 & 77.1892 & 65.969598 & 91.646881 \\
\hline & & 63.188252 & 81.513418 & 63.101017 & 88.917082 & 63.558405 & 76.831445 & 66.076159 & 91.887804 \\
\hline \multicolumn{10}{|c|}{ Symmetric fold type II $\left(\alpha=0.00001, \theta_{\text {init }}=179.43028^{\circ}\right)$} \\
\hline$\lambda_{1}$ & 0.75 & \multicolumn{4}{|c|}{98.601081} & \multicolumn{4}{|c|}{98.502297} \\
\hline$\lambda_{2}$ & 0.865 & \multicolumn{2}{|c|}{93.588105} & \multicolumn{2}{|c|}{109.48} & \multicolumn{2}{|c|}{93.037603} & \multicolumn{2}{|c|}{106.61775} \\
\hline$\lambda_{3}$ & 0.89225 & \multirow{2}{*}{\multicolumn{2}{|c|}{$\begin{array}{l}92.307411 \\
92.525161\end{array}$}} & \multicolumn{2}{|c|}{105.01353} & \multicolumn{2}{|c|}{93.787106} & \multicolumn{2}{|c|}{112.10937} \\
\hline & & & 92.525161 & \multicolumn{2}{|c|}{102.82903} & \multicolumn{2}{|c|}{94.351735} & \multicolumn{2}{|c|}{13.5141} \\
\hline \multirow[t]{2}{*}{$\lambda_{4}$} & 0.8925 & 92.302608 & 105.23 & 97.734594 & 111.98573 & 92.541349 & 102.72656 & 94.379436 & 113.5926 \\
\hline & & 92.33191 & 105.96831 & 92.267878 & 111.53614 & 92.603746 & 102.45876 & 94.458098 & 113.77415 \\
\hline
\end{tabular}

considering the natural data in relation to stress and the changes in the fold morphologies in a temporal domain. Such a justification explains whether the HDSFs will change its phases. Periodic locking is observed at the $\mu$ values between 0.848 and 0.860 (Table V).

This analysis is shown to have better understanding that this data is following deterministic randomness, that is each successive value depends on the value of its predecessor. The time dependent stress dynamical system is also represented as return $\operatorname{maps}\left(\lambda_{t+1}-\lambda_{t}\right.$ vs $\left.\lambda_{t+2}-\lambda_{t+1}\right)$ for the $\mu=0.9,0.95$ and 0.9925 . Figure 5(a)-(c) illustrates these return maps. These illustrations allow for qualitative understanding of the stress dynamics that follow the deterministic randomness.

\section{Period Locking}

Period locking is identified between the dynamics of the stress regulatory parameter and the dynamics of the fold system. From the fold dynamics that is being controlled by time dependent stress regulatory parameter, one can see that the dynamics of the time dependent stress regulatory parameter is enslaved to the dynamics of the fold system. The dynamics of stress regulatory parameter is following period two, however, the dynamics of the fold system that is being controlled by this controlled stress dynamics follows the period 4. This "period locking" is observed between the $\mu$ the stress regulatory parameter in the modulated logistic system, values 0.848 and 0.860 . This possibility of the periodic locking in the modulated fold dynamical system needs to be described by analyzing the physical forces of specific range. This needs to be compared in a meaningful way with the stress regulatory control parameter represented as a numerical value (i.e., $\mu<1>0$ ). It is interesting to see how the dynamics of the stress regulatory parameter is enslaved to the dynamics of fold morphological behavior between the values of 0.848 and 0.860 (Table V).

\section{Bifurcation Diagrams}

\section{Fold Dynamics Under the Influence of Constant Stress}

In Fig. 7(a), a bifurcation diagram is shown for various possible dynamical behaviors of the symmetric folds under dynamics, viz, stable, unstable, chaotic. The evolution types of fold transformations can be segregated as period zero, period one, period two, and chaotic. As the parameter $\lambda$ is varied, changes in the qualitative behaviour of the system 


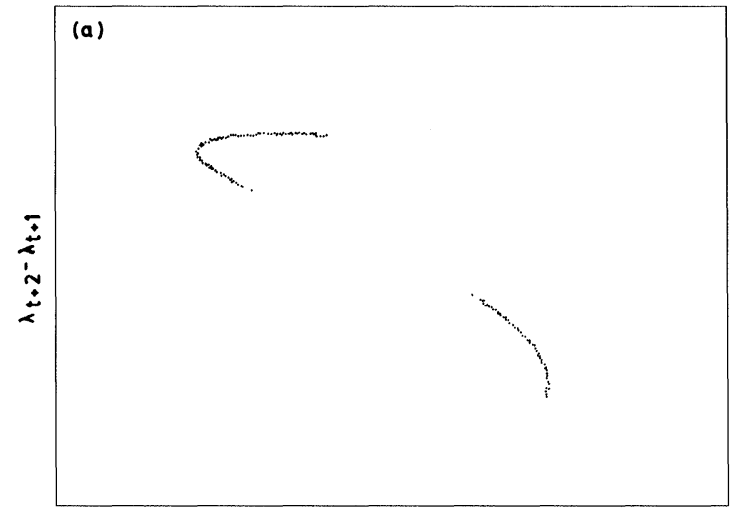

$\lambda_{t+1}-\lambda_{t}$

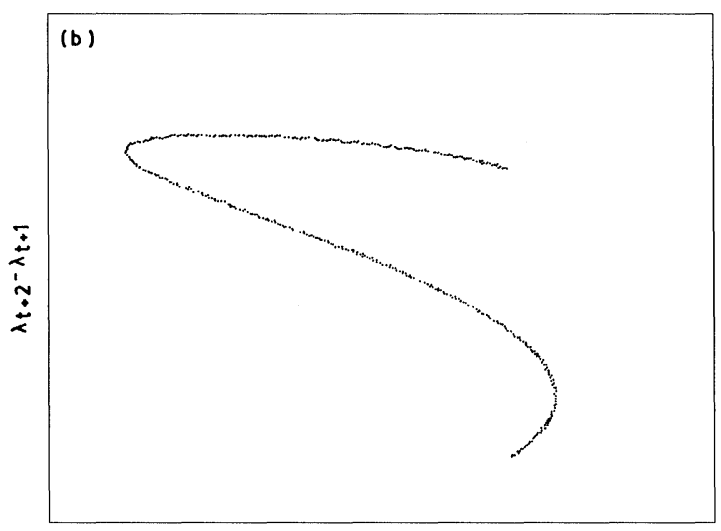

$\lambda_{t+1}-\lambda_{t}$

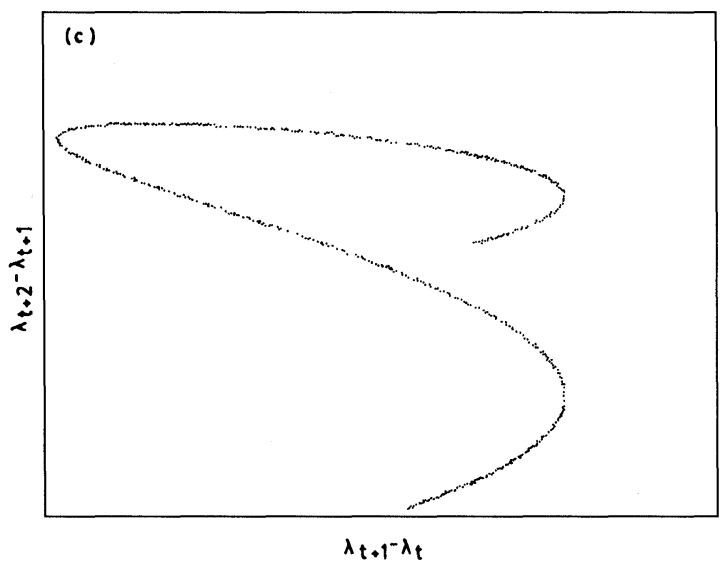

FIGURE 5 Return map of the dynamics of time dependent stress regulatory parameter $\left(\lambda_{t+1}-\lambda_{t}\right.$ vs $\left.\lambda_{t+2}-\lambda_{t+1}\right)$. The stress modulation control parameter $(\mu)$ that controls the time dependent stress regulatory parameter, (a) $\mu=0.9$, (b) 0.95 and (c) 0.9925 . The initial normalized stress parameter $\lambda_{1}=0.00001$.

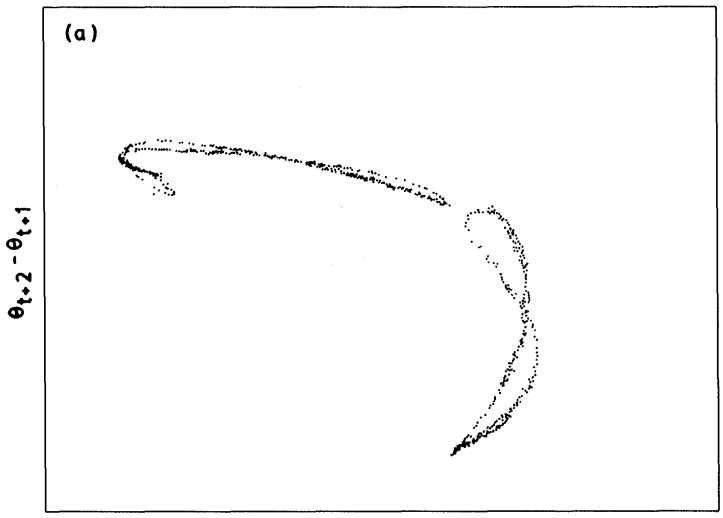

$\theta_{t+1}-\theta_{t}$

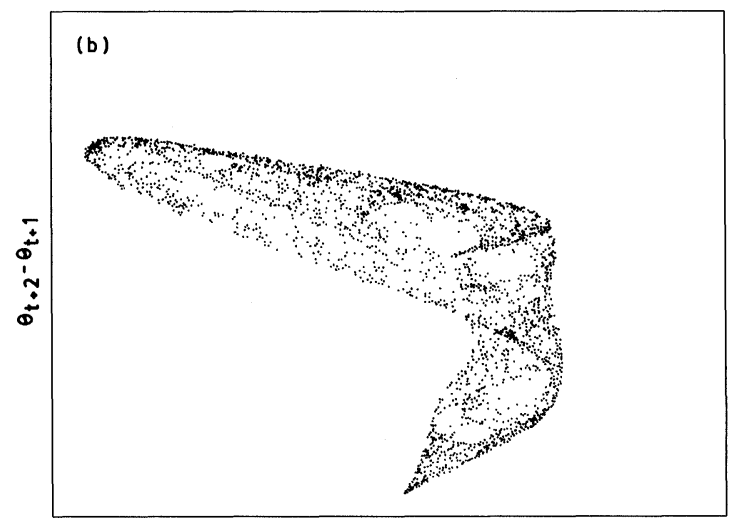

$\theta_{t+1}-\theta_{t}$

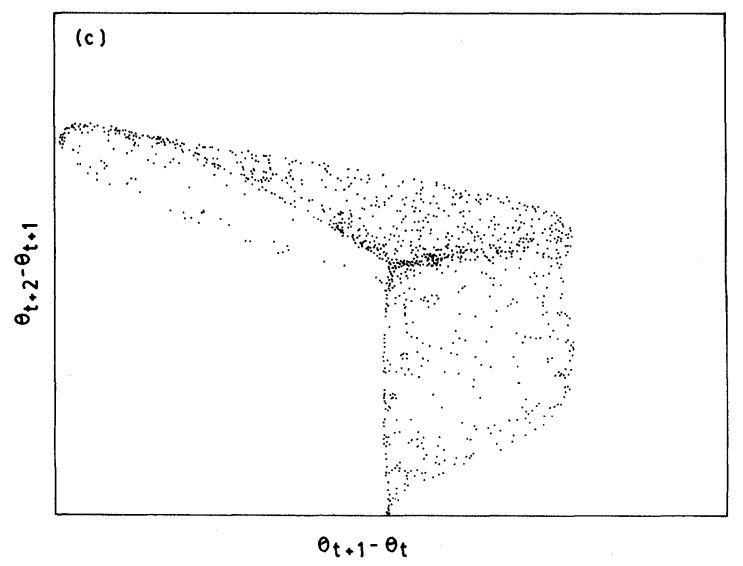

FIGURE 6 Return map of the modulated fold morphological dynamics by time dependent stress regulatory parameter $\left(\theta_{t+1}-\theta_{t}\right.$ vs $\left.\theta_{t+2}-\theta_{t+1}\right)$. The control parameter (a) $\mu=0.9$, (b) 0.95 and (c) 0.9925 . The specifications for the simulation are: $\lambda_{t}=0.00001, \theta_{t}=179.43028^{\circ}$ or $\alpha_{t}=0.00001$ and iteration no. $10 \times 10^{6}$ time steps. This map is plotted in $\theta$-parameter space. 
TABLE IV AIAs at the threshold regulatory forces after $3 \times 10^{4}$ time steps: The dynamical rule is simple first order nonlinear difference equation with time dependent stress control parameter

\begin{tabular}{|c|c|c|c|c|c|c|c|c|c|}
\hline \multicolumn{2}{|c|}{$\begin{array}{l}\text { Threshold control } \\
\text { parameter }\end{array}$} & \multicolumn{8}{|c|}{ AIAs (deg.) } \\
\hline \multicolumn{10}{|c|}{ Symmetric fold type I $\left(\alpha=0.00001, \theta_{\text {init }}=179.43028^{\circ}, \lambda=0.00001\right)$} \\
\hline$\mu_{1}$ & 0.75 & \multicolumn{4}{|c|}{73.327164} & \multicolumn{4}{|c|}{73.728954} \\
\hline$\mu_{2}$ & 0.865 & \multicolumn{2}{|c|}{107.68174} & \multicolumn{2}{|c|}{64.294614} & \multicolumn{2}{|c|}{82.599485} & \multicolumn{2}{|c|}{77.825268} \\
\hline \multirow[t]{2}{*}{$\mu_{3}$} & 0.89225 & \multicolumn{2}{|c|}{80.778449} & \multicolumn{2}{|c|}{84.660144} & \multicolumn{2}{|c|}{115.27091} & \multicolumn{2}{|c|}{63.503065} \\
\hline & & \multicolumn{2}{|c|}{79.025155} & \multicolumn{2}{|c|}{88.477219} & \multicolumn{2}{|c|}{118.38197} & \multicolumn{2}{|c|}{63.266815} \\
\hline \multirow[t]{2}{*}{$\mu_{4}$} & 0.8925 & 80.991449 & 84.305693 & 114.9721 & 63.528255 & 78.985088 & 88.734899 & 118.60113 & 63.24672 \\
\hline & & 81.746702 & 83.107759 & 113.92723 & 63.613464 & 78.985146 & 89.642031 & 119.39317 & 63.158243 \\
\hline \multicolumn{10}{|c|}{ Symmetric fold type II $\left(\alpha=0.00001, \theta_{\text {init }}=179.43028^{\circ}, \lambda=0.00001\right)$} \\
\hline$\mu_{1}$ & 0.75 & \multicolumn{4}{|c|}{99.840737} & \multicolumn{4}{|c|}{100.14045} \\
\hline$\mu_{2}$ & 0.865 & \multicolumn{2}{|c|}{125.68524} & \multicolumn{2}{|c|}{93.145029} & \multicolumn{2}{|c|}{106.7839} & \multicolumn{2}{|c|}{103.20273} \\
\hline$\mu_{3}$ & 0.89225 & \multicolumn{2}{|c|}{105.41666} & \multicolumn{2}{|c|}{108.33262} & \multicolumn{2}{|c|}{131.40715} & \multicolumn{2}{|c|}{92.563091} \\
\hline & & \multicolumn{2}{|c|}{104.10171} & \multicolumn{2}{|c|}{111.20491} & \multicolumn{2}{|c|}{133.75145} & \multicolumn{2}{|c|}{92.389588} \\
\hline \multirow[t]{2}{*}{$\mu_{4}$} & 0.8925 & 105.57651 & 108.06612 & 131.18194 & 92.581572 & 104.07168 & 111.39894 & 133.91655 & 92.374946 \\
\hline & & 106.14345 & 107.16576 & 130.39438 & 92.644198 & 104.07172 & 112.08213 & 134.51323 & 92.309882 \\
\hline
\end{tabular}

TABLE V AIAs of the fold dynamical system following time dependent stress control parameters $\left(\lambda_{0}=0.00001 ; \alpha_{0}=0.00001\right.$ or $\theta_{t}=179.43028^{\circ}$; No. of iterations $3 \times 10^{4}$ )

\begin{tabular}{lcccc}
\hline $\begin{array}{l}\text { Stress modulation parameter }(\mu) \\
\text { to control time dependent stress dynamics }\end{array}$ & \multicolumn{2}{c}{$\lambda_{t+1}=4 \mu \lambda_{t}\left(1-\lambda_{t}\right)$} & \multicolumn{2}{c}{ AIAs $\left(\theta^{*}\right)$} \\
\cline { 2 - 3 } \cline { 5 - 5 } & Attractor time dependent stress control parameters $\left(\lambda^{*}\right)$ & & Fold type I & Fold type II \\
\hline 0.848 & 0.453991 & 92.389892 & 114.15255 \\
& & 41.528587 & 95.894165 \\
& 0.84082 & 92.3629 & 114.13221 \\
0.860 & & 41.563547 & 95.903425 \\
& 0.442194 & 96.82515 & 117.49648 \\
& & 66.579149 & 94.829506 \\
& 0.848505 & 89.600981 & 112.05121 \\
& & 70.063627 & 97.411428 \\
\hline
\end{tabular}

can occur. Such qualitative behavior can be seen in the bifurcation diagram (Fig. 7(a)) in which the attractor set against control parameter $\lambda$ is plotted. In this bifurcation diagram, as $\lambda \in[0,1]$, the dynamical behavior possesses one stable fixed point. As $\lambda$ is increased past 0.75 , the behavior becomes unstable and two new stable periodic points appear. Fold behavior follows periodicity where both amplification and shortening of folds are subsequently involved. The dynamics become unstable, each originating two new stable periodic points of period 4 as $\lambda$ is further increased from 0.89225 . Through this bifurcation diagram, the fold dynamical behavior path can be found with respect to the control parameters. This diagram (Fig. 7(a)) not only portrays the type of dynamical behaviors of the fold with respect to the control parameter, but also the critical states in terms of IAs of the fold under dynamics. The number of critical states that a fold reaches under the dynamics depends on the initial fold state and the control parameter $(\lambda)$. For every value of $\lambda$, there will be an attracting point. These attracting points are represented in two ways: (a) the fractal dimension in normalized scale and (b) critical states shown as IA of a symmetric fold under dynamics. Instead of the fractal dimensions in normalized scale, their corresponding IAs are represented. If the fold under dynamics is according to the rule of the first order difference equation (2), Fig. 7(a) shows various behavior paths and their 


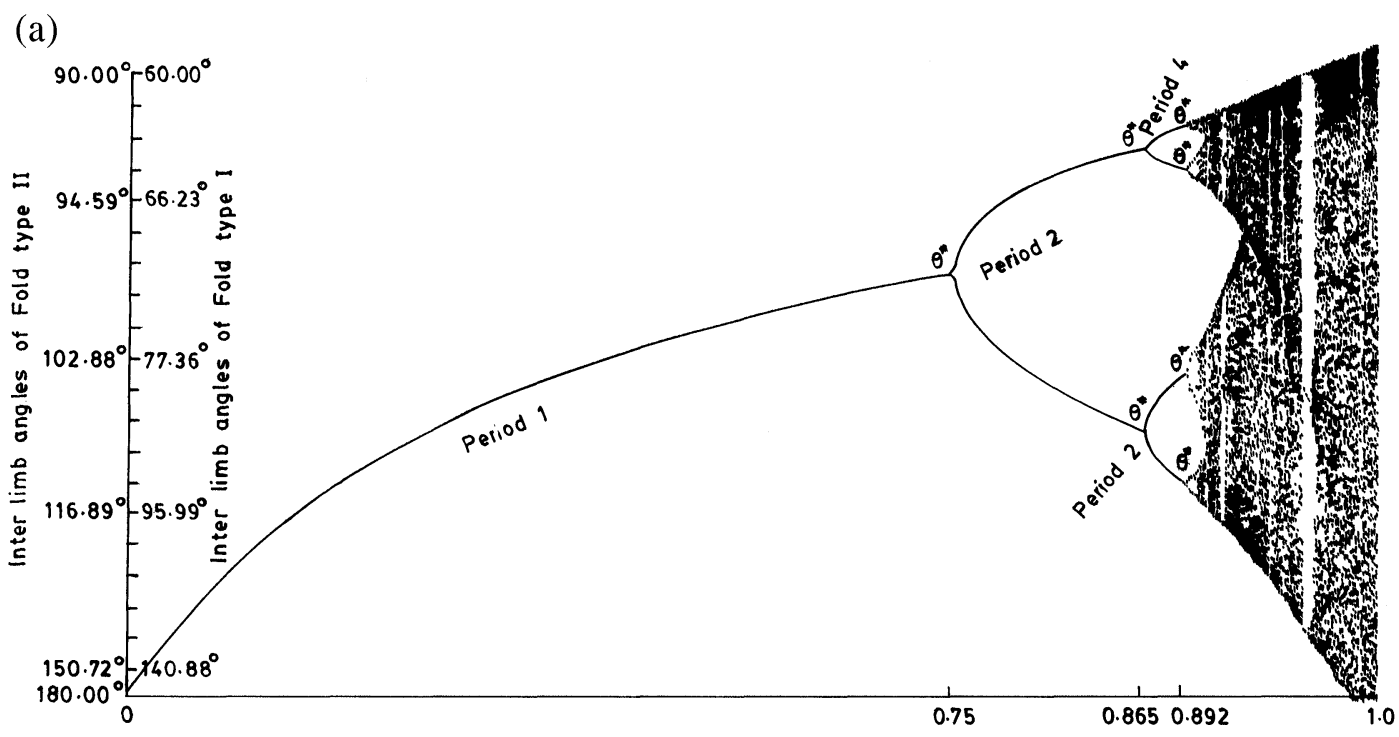

Constant stress control parameter $(\lambda)$

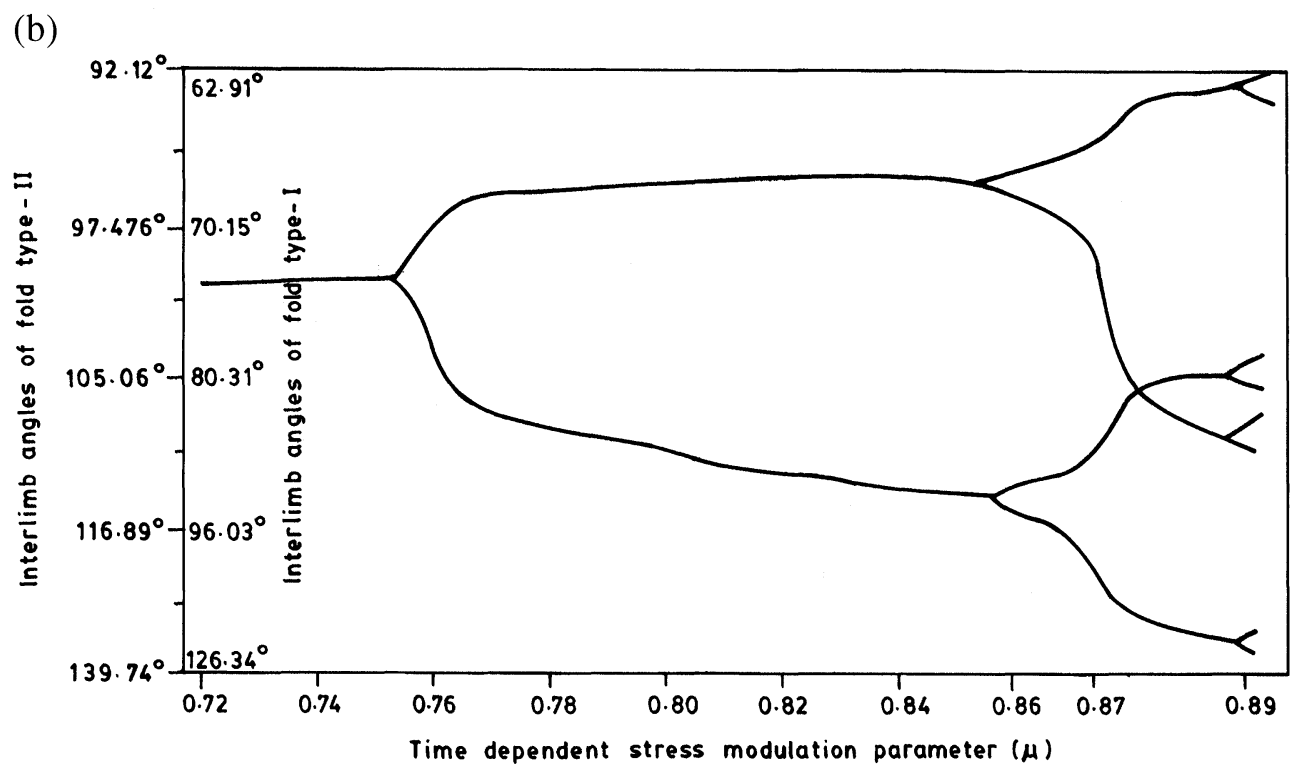

FIGURE 7 (a) Bifurcation diagram showing various possibilities of fold transformations: stable, unstable, chaotic. The value of $\lambda$ measures the constant strength of stress regulatory parameter which controls the fold. The evolution of the fold system can be segregated as period zero, period one, period two, and chaotic. Period zero: A contorted fold with $\alpha_{t}=0.06363$ becomes straight when $\alpha_{t+1}$ approaches zero, $\lambda$ is between 0 and 0.25 . This is possible under the process of continuous fold amplification. Period one: When $\lambda$ is between 0.25 and 0.75 , the fold pattern shortens, and the pattern reaches a fixed point attractor. It means that the fold reaches the equilibrium state. Period two: The fold pattern oscillates between two points when $\lambda$ is between 0.75 and 0.89225. The fold amplification and compression will occur periodically. Chaotic: The behavior of fold is such that the fold shapes at different time periods do not overlap. Here, the fold amplification and compressions may occur, as time progresses, randomly. The values on both sides of the $Y$-axis represent the IAs of the symmetric folds with three limbs and two limbs respectively. (b) Bifurcation diagram of Eq. (11) that describes the fold dynamics under the influence of time dependent stress regulatory parameter. The branches crossing over each other in the 4-cycle region result in a complete modification of the structure. 
stability with respect to the initial fold state and $\lambda$ values were shown by means of respective critical states represented by $\theta$ 's. The important contribution of this diagram (Fig. 7(a)) is the information regarding the history of folding that can be studied, provided the initial state of the symmetric fold and the control parameter that controls the fold dynamics are precisely computed.

\section{Dynamical System Under the Influence of TDSD}

The influence of TDSD on fold dynamical behavior is depicted through bifurcation diagram (Fig. 7(b)). This is controlled by considering Eqs. (12) and (14). In these equations, the value of the parameter $\lambda$ at any instant is a single nonlinear function of its value in the previous instants. In this, $\mu$ plays the role of the control parameter, which is thought of as the strength of stress modulated parameter.

For $0<\mu<0.75$, stress dynamics follows attracting fixed point for $\lambda_{t}$ and here for $\theta_{t}, \theta_{t}\left(180^{\circ}, 60^{\circ}\right.$, $\left.90^{\circ}\right)$ and $\lambda \in[0,1]$. The bifurcation diagram in Fig. 7(b) is generated by starting from a parameter value $\mu=0.75$ and increase it in steps of 0.001 , by an initial values of $\theta_{t}$ and $\lambda_{t}$, say 179.43028 and 0.00001 , respectively.

Due to modulation by TDSD, changes between the bifurcation diagrams (Fig. 7(a) and (b)) are observed. The fundamental difference is that the bifurcation occurs earlier than in the case of the fold dynamics under the influence of constant stress dynamics from the observed bifurcation orders. The normal feature in the modulated system is the crossing-over of the inner bifurcation branches in the 4-cycle region. It lacks the symmetry of the bifurcation structure of the fold dynamical systems that is influenced by the CSD.

\section{CONCLUSIONS}

We have studied the highly ductile nature of symmetric fold dynamical behaviors that are controlled by the constant and the time dependent stress modulated parameters respectively through numerical simulations. In particular we discuss the computations of the changing AIAs at respective stress modulated parameters which are used to control the behavior of fold dynamical system. Equations are proposed to compute IA of these symmetric folds undergoing dynamical changes, which encompass the rule that is ensued to transform the folds and certain specifications of the folds. Bifurcation diagrams are described to show how these symmetric folds under dynamics behave under the change of constant stress control parameter, $\lambda$, and the strength of stress modulated parameter, $\mu$, to control TDSD, $\lambda_{t}$. The AIAs $\left(\theta^{*}\right)$ are shown on the bifurcation diagrams. By considering these AIAs, equations are also proposed to compute metric universality. The periodic nature of the phase changes in the fold morphological dynamics is studied using the time dependent and constant stresses that follow a dynamical rule. Interesting conclusions are arrived at in terms of variations in the AIAs of the fold following these two dynamical rules. These theoretical conclusions have an important bearing when considering strategies for the understanding of geological fold dynamics; more generally, when considering the behaviors of natural time series data in a range of geological situations where folding is taking place. This type of time series data indicate that the possibility of predicting predictability depends on the degree of randomness in the behavior of the dynamical system. From the time series data, attractor can be constructed in phase space. The dimension of the attractor provides the possibility of predicting predictability. Low dimensional attractors of dynamical systems allow the behavior to be predicted through some nonlinear equations. However, as the dimensionality of the attractor that describes the behavior of dynamical system is high, the predictability becomes difficult. These two types of systems are termed as the dynamical systems that follow deterministic randomness and the natural randomness in their behaviors. Generally, the system that follows deterministic randomness will possess the strange attractor of which the dimensionality is low. The assumptions considered as the bases are that the dynamics of both fold morphology and the acting 
stress possess the low dimensional attractors. To infer whether the attractor of fold morphological dynamics posesses the low dimensionality, a long time series data is required.

This deterministic approach emphasizes to give certain possible behaviors of fold dynamics with the respective critical states represented by IAs. It is concluded that the critical states of symmetric folds under dynamics depend on the stress that influences the fold, and the initial state of the fold. With the aid of the SSM parameter and the specifications of initial state of symmetric fold, graphic analysis may be carried out to investigate the history of folding. Such an investigation, to find out the critical states of several possible behaviors, will shed light on predicting the fold dynamical behaviors. The dynamically transforming symmetric fold with different time dependent and constant stress controlling parameters was shown for a better qualitative understanding. This qualitative study is an attempt as an example for academic interest to furnish the interplay between numerical experiments and analytical theory. This maiden attempt is considered as a preliminary effort to introduce bifurcation theory for the understanding of the dynamical behavior of symmetric folds. In brief, this paper presents a maiden attempt to show how a symmetric fold can modify its shape, in particular the interlimb angle, through a nonlinear first order difference equation. This approach could be valid as a potential application of these equations to a geological problem to resolve real fold cases. However, with historical data available, the phase that the fold has undergone can be studied by investigating the fold at different time intervals to fit the equations. From such derived equations, assumed to be first order nonlinear difference equations, as the underlying dynamical rule in the present qualitative investigation, our understanding of the fold dynamics will certainly be enhanced.

\section{Acknowledgments}

The author is grateful to Sir Robert M. May, F. R. S., for his encouragement, and to Dr. Juan I. Soto for his invaluable suggestions and constructive criticism on the earlier version of this work. The author is also grateful to Prof. V. R. R. M. Babu for stimulating discussions. The financial support given by Department of Science and Technology, under grant no. SR/SY/A-06/94, and a research associate fellowship from Council of Scientific and Industrial Research, India, are thankfully acknowledged.

\section{References}

M. Chapple (1968). A mathematical theory of finite amplitude folding. Geol. Soc. Ame. Built., 79, 47-68.

J.H. Dieterich and N.L. Carter (1969). Stress history of folding. Ame. Jour. of Scie., 267, 129-154.

J.H. Dieterich (1970). Computer experiments on mechanics of finite amplitude folds. Canad. Jour. of Eart. Scie., 7, 467-476.

D.K. Parrish (1973). A nonlinear finite element fold model. Ame. Jour. of Scie., 273, 318-334.

W.D. Means (1976). Stress and Strain. Basic Concepts of Continuous Mechanics for Geologists. Springer Verlag, New York, p. 339.

W.D. Means (1990). Kinematics, stress, deformation and material behaviour. Journal of Structural Geology, 12, 953971.

J.G. Ramsay and M.I. Huber (1987). The techniques of modern structural geology. Vol. 2: Folds and Fractures, Academic Press, London, p. 391.

N.J. Price and J.W. Cosgrove (1990). Analysis of Geological Structures, Cambridge University Press, Cambridge, p. 502.

T.R. Maden (1983). Microcrack connectivity in rocks: a renormalization group approach to the critical phenomena of conduction and failure in crystalline rocks. Journal of Geophysical Research, 88, 585-592.

C.J. Allegre, L. Le Mouel and A. Provist, A (1982). Scaling rules in rock fracture and possible implications for earthquake prediction. Nature, 297, 47-49.

W.I. Newman and L. Knopof (1982). Crack fusion dynamics: a model for large earthquakes. Geophysical Research Letters, 9 , 735-738.

R.F. Smalley, Jr., D.L. Turcotte and S.A. Solla (1985). A renormalization group approach to study stick-slip behavior. Journal of Geophysical Research, 90, B2, 1894-1900.

P. Davy, A. Sornette and D. Sornette (1990). Some consequences of a proposed fractal nature of continental faulting. Nature, 348, $56-58$

B.B. Mandelbrot (1982). Fractal Geometry of Nature, Freeman \& Co., San Francisco, p. 468

R.M. May (1976). Simple mathematical models with very complicated dynamics. Nature, 261, 459-467.

B.E. Hobbs, W.D. Means and P.F. Williams (1976). An Outline of Structural Geology. John Wiley \& Sons, New York.

D. Ruelle (1987). Diagnosis of dynamical system with fluctuating parameters. Proc. R. Soc. Lon., A413, 5-8.

M. Feignbaum (1981). Universal behavior in nonlinear systems. Los Alamos Scien., 1, 4-27. 


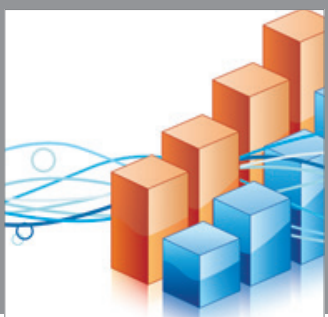

Advances in

Operations Research

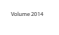

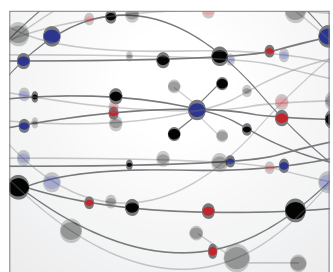

\section{The Scientific} World Journal
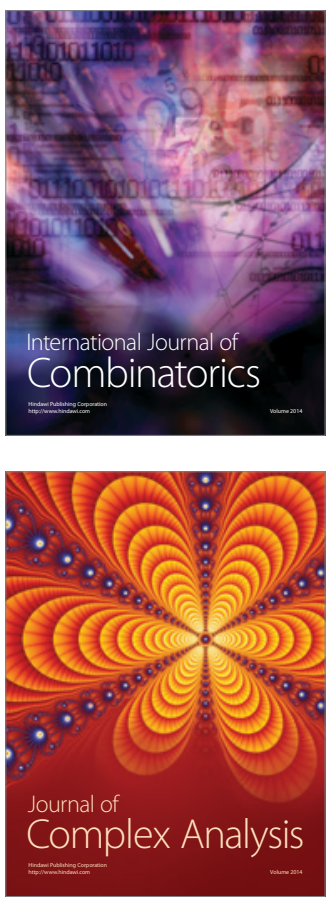

International Journal of

Mathematics and

Mathematical

Sciences
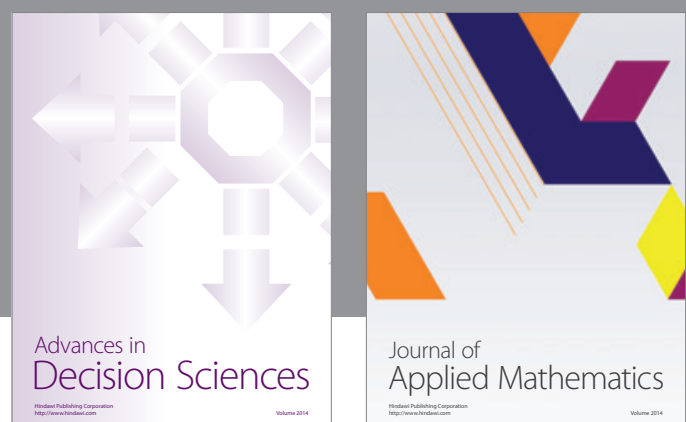

Journal of

Applied Mathematics
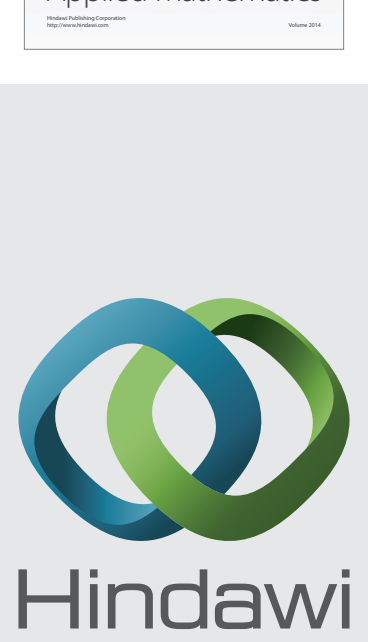

Submit your manuscripts at http://www.hindawi.com
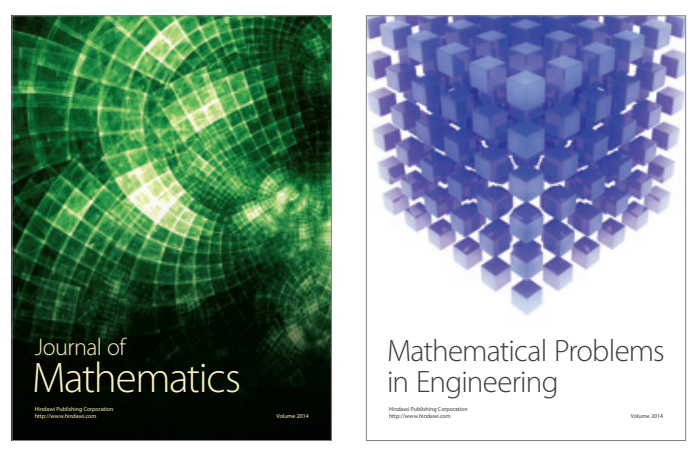

Mathematical Problems in Engineering
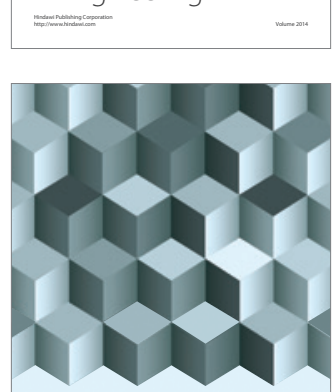

Journal of

Function Spaces
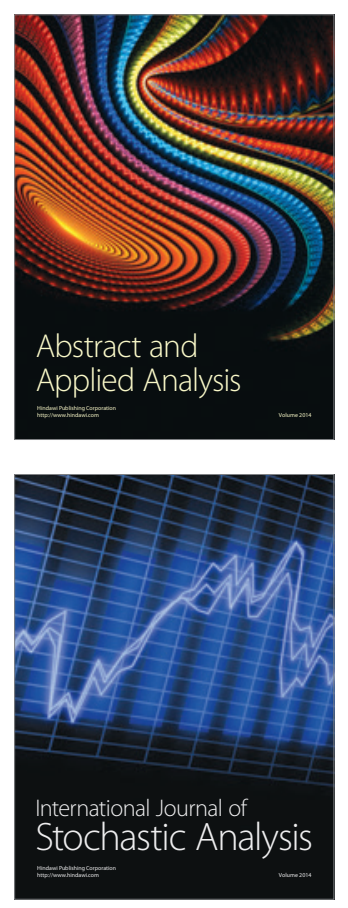

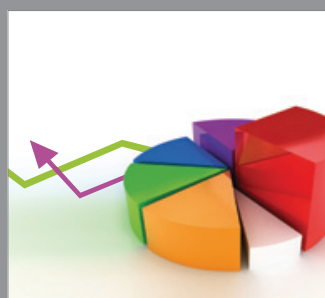

ournal of

Probability and Statistics

Promensencen
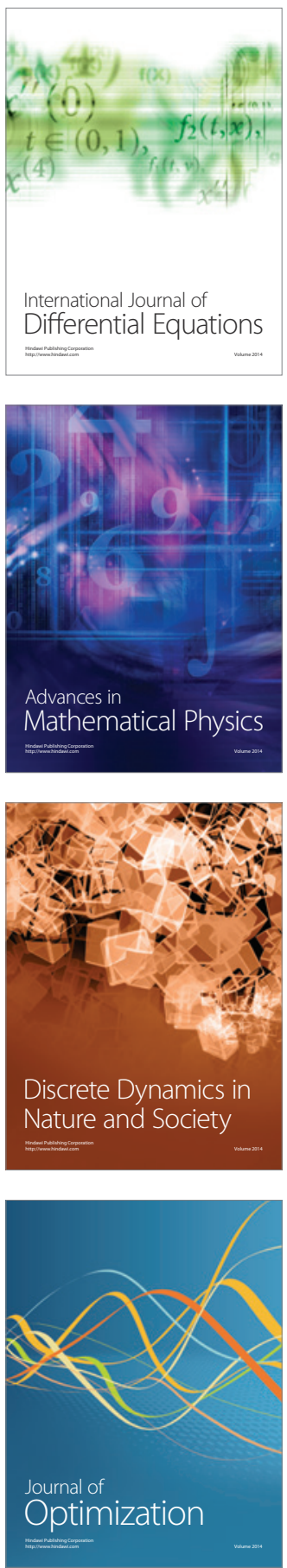